\section{Common toxicities}

\section{Sophie McMurrough}

Toxic substances surround our patients everyday but how much do we know about the common toxins we see in practice? Toxicities can present with a variety of clinical signs from neurological deficits, renal injuries and coagulopathies! In this talk we will discuss what happens to the body when intoxicated and learn how to manage each case independently.

\section{POISON HELPLINES AND USEFUL INFORMATION}

- Veterinary Poisons Information Service (VPIS) is a global 24-hour emergency helpline which provides information on a huge variety of toxins. VPIS Animal Poison Line: 01202509000

- Vets Now Tox Box works alongside VPIS providing 24-hour access to the antidotes required for many toxicities. These are stocked for collection in most Vets Now clinics across the UK

- Make your own toxin log, documenting details of toxins and the information gained from these services will give you free and instant access in practice

- BSAVA Poisons Database is also a useful tool for members to search for specific toxins, look up the clinical signs and treatment options.

\section{EMERGENCY TRIAGE (PHONE OR ON PRESENTATION)}

A very important role of the veterinary nurse is emergency triage. We need to determine if the pet needs to see a vet. If so, how soon? Is it an emergency?

\section{NON-STEROIDAL ANTI-INFLAMMATORY DRUGS}

Non-steroidal anti-inflammatory drugs (NSAIDs) are readily available and can be particularly toxic to dogs causing vomiting, diarrhoea, gastric (Gl) ulceration and acute kidney injury (AKI).

\section{Clinical signs}

- Anorexia

- Vomiting/haematemesis

- Diarrhoea/melaena

- Polyuria/polydipsia

- Oliguria/anuria.

\section{Initial treatment}

Induce emesis and administer multiple doses of activated charcoal. Gastroprotectants and anti-emetics should be administered. Aggressive intravenous fluid therapy for the treatment of $\mathrm{AKI}$ and close monitoring of urine output with the use of in-dwelling urinary catheters.

\section{ETHYLENE GLYCOL (ANTIFREEZE)}

Ethylene glycol is not toxic, but its metabolites cause a severe toxicosis. Antidote therapy is to prevent the metabolism of the dangerous metabolites by the liver This toxicity can cause metabolic acidosis, cardiopulmonary failure, central nervous system depression and AKI.

Clinical signs (30 minutes after ingestion):

- Ataxia

- Depression

- Hypersalivation

- Vomiting

- Polyuria/polydipsia.

Clinical signs of secondary AKI (24-72 hours after ingestion):

- Anorexia

- Depression

- Oliguria/anuria

- Ureamic halitosis

- Oral ulceration.

\section{Initial treatment}

Therapy to prevent the metabolism of ethylene glycol must be initiated within 3 hours of ingestion in cats and 8-12 hours in dogs. If treatment is delayed for longer than 3 hours in cats, the risk of fatality is almost $100 \%$.

\section{KEY LEARNING OBJECTIVES}

- Learn about a variety of common toxicities seen in small animal practice

- Understand the physiology of each toxin

- Learn key nursing considerations and treatment of the toxicities discussed

\section{MULTIPLE CHOICE QUESTIONS}

1. Which two systems are most commonly affected by NSAID toxicity?

(A) Hepatic and nervous

(B) Gastrointestinal and renal

(C) Gastrointestinal and hepatic

(D) Renal and hepatic

2. Ethylene glycol antidote treatment should be initiated within how many hours of exposure in the cat?
(A) 8 hours
(B) 12 hours
(C) 3 hours
(D) No time limit

3. Ethylene glycol is metabolized by what organ(s)?
(A) Liver
(B) Kidneys
(C) Gastrointestinal tract
(D) Brain 